\title{
EFFECTS OF TEN MEDICINAL PLANTS ON SERUM GLUCOSE LEVELS, LIPID PARAMETERS AND LIVER ENZYMES IN EXPERIMENTAL DIABETES
}

\author{
(C) Montasser Kouhsari Sh. 1, Barahoui S. 1, Morshedzadeh F. ${ }^{2}$, Panahandeh P. 1, \\ Safa Issini M. ${ }^{2}$, Sotoudeh Haghighi Sh. ${ }^{2}$, Monavarfeshani A. ${ }^{1}$
}

${ }^{1}$ Department of Cellular and Molecular Biology, School of Biology, University College of Science, University of Tehran, Iran ${ }^{2}$ Department of Cellular and Molecular Biology, Kish International Campus, University of Tehran, Kish, Iran

Diabetes mellitus is a common and serious health problem worldwide, and hence, any investigation in this area plays a crucial role in the improvement and treatment of patients. Actually, in many countries and also in Iran, more than 800 medicinal plants are used as traditional remedies for the treatment of diabetes.specially because, common antidiabetic drugs have many inconvenient side effects. In this study we have investigated the effects of 10 different plants extracts, Camellia sinensis, Galega officinalis, Laurus nobilis, Cucurbita pepo, Nastrurtium officinale, Olea europaea, Otostegia persica, Pistacia vera, Punica granatum and Rosa canina, on diabetic male Wistar rats in comparision with antidiabetic common refrence drugs, such as, Acarbose, Metformin and Glybenclamide. Aquous, ethanolic and methanolic extracts of 10 different medicinal plants were prepared and administrated, at dose of $250 \mathrm{mg} / \mathrm{kg}$ bw, to Alloxan monohydrate diabetic male Wistar rats. The effects of different plants extracts on plasma glucose levels were measured, in short and long term periods, by enzymatic assay (Glucose Oxidase). Also, plasma lipids, TC, TG, HDL, LDL, VLDL, and liver enzymes, AST (Aspartate aminotransferase), ALT (Alanine aminotransferase) and ALP (Alkaline phosphatase) concentrations were evaluated enzymatically. Different plants extracts showed significant reduction in PBG (postprandial blood glucose). Also some of them proved considerable changes in the levels of plasma lipid profiles and liver enzymes.

\section{CHROMATOGRAPHY COMBINED WITH BIOASSAYS AND OTHER HYPHENATIONS - THE DIRECT LINK TO THE COMPOUND INDICATING THE EFFECT}

\section{(C) Morlock Gertrud E.}

Chair of Food Science, Justus Liebig University Gie en, Heinrich-Buff-Ring 26, 35392 Gießen, Germany

Initially advocated by T. Hirschfeld, the term hypernation (super-hyphenation) was coined to place all of the required spectrometers into a single system so that all of the spectroscopic information is obtained in a single run (1). Hypernation represents a logical, rapid and efficient strategy for obtaining the maximum possible information out of a single separation. Problems associated with column-based super-hyphenations are much less challenging in planar chromatography-based hyphenations: The open planar system is highly adaptive to different sensitivities, cost-effective by modular instrumentation compared to the status quo in analysis, generating less data due to targeted access to points-of-care on the plate, and directly accessible for the respective optimal detector solvent. The eluent is evaporated after high-performance thinlayer chromatography (HPTLC) and not impacting the different detectors. The latter is extremely relevant for effect-directed detection with bioassays. HPTLC is highly compatible to the direct application of bioassays onto the chromatogram and can be the direct link to the compound indicating the effect, especially after elution of the zone of interest into a high-resolution mass spectrometer. Existing super-hyphenations are for example (2):

- HPTLC/UV/Vis/FLD/MS

- HPTLC/UV/Vis/FLD/bioassay/HRMS

- HPTLC/UV/Vis/FLD/FTIR ATR

Examples are given in the field of natural product search and food analysis (3-7).

References: (1) I.D. Wilson, Th. A. Brinkman, TrAC 26/9, 2007, 847-854. (2) G. Morlock, W. Schwack, TrAC 29/10, 2010, 1157-1171. (3) A. Klöppel, W. Gasse, F. Brümmer, G. Morlock, J. Planar Chromatogr. 21, 2008, 431-436. (4) G. Morlock, C. Oellig, J. AOAC Int., 2009, 745756. (5) Morlock, G., Gamlich, F. J. Planar Chromatogr., 2012, in print. (6) Misra, K., Tulsawani, R., Shyam, R., Kumar Meena, D., Morlock, G., J. Liq. Chromatogr. Relat. Technol., 2012, in print. (7) Morlock, G., Y. Sung, B. Honermeier, in submission. 